\title{
Atenção farmacêutica e práticas integrativas e complementares no SUS: conhecimento e aceitação por parte da população sãojoanense
}

\section{I ${ }^{1}$ Luciene Alves Moreira Marques, ${ }^{2}$ Flávia Vanessa Vieira Ribeiro do Vale, ${ }^{3}$ Valéria Aparecida dos Santos Nogueira, ${ }^{4}$ Fábio Luiz Mialhe, ${ }^{5}$ Lara Cristina Silva I}

Resumo: Em 2006, o Ministério da Saúde criou a Política Nacional de Terapias Integrativas e Complementares no SUS. O objetivo do estudo foi investigar o conhecimento e a aceitação das terapias integrativas e complementares e atenção farmacêutica por parte dos usuários do SUS. O estudo foi realizado na farmácia da Unidade Básica de Saúde da cidade de São João da Mata, Minas Gerais, Brasil. Um estudo transversal descritivo qualitativo e quantitativo foi realizado com três médicos e 35 usuários do serviço. Destes, 100\% não conheciam as terapias integrativas e complementares. Após explicação clara e simples pelo pesquisador, 31,42\% disseram que aceitariam o uso de fitoterapia, 51,42\% aceitariam a acupuntura, 37,14\% aceitariam a homeopatia e nenhum utilizaria a crenoterapia. Quando questionados sobre a atenção farmacêutica, 45,71\% disseram já ter ouvido falar neste assunto, 22,85\% sabem do que se trata e 31,42\% nunca tinham ouvido falar em atenção farmacêutica. Quando os três médicos que atendem na unidade de saúde foram questionados, observou-se indiferença, não-aceitação e aceitação, respectivamente. Em conclusão, este estudo demonstrou que a grande maioria dos pesquisados aceitaria as terapias integrativas e complementares se estas fossem oferecidas pela unidade de saúde. Além disso, os usuários acham importante uma maior atuação do farmacêutico. É necessária a implantação de programas de divulgação para os pacientes e principalmente para os médicos prescritores de práticas integrativas e complementares.

> Palavras-chave: atenção farmacêutica; terapias integrativas e complementares; aceitação.

\author{
1 Professora de Atenção \\ Farmacêutica da Faculdade \\ de Ciências Farmacêuticas \\ da UNIFAL-MG. Endereço \\ eletrônico: lualvesmarques@ \\ yahoo.com.br \\ ${ }^{2}$ Especialista em Atenção \\ Farmacêutica pela UNIFAL- \\ MG. Endereço eletrônico: \\ lumarques@unifal-mg.edu.br \\ ${ }^{3}$ Doutoranda em Saúde \\ Coletiva pela FOP-UNICAMP. \\ Endereço eletrônico: vasanog@ \\ fop.unicamp.br \\ ${ }^{4}$ Professor Doutor de Saúde \\ Coletiva, FOP-UNICAMP. \\ Endereço eletrônico: mialhe@ \\ fop.unicamp.br \\ ${ }^{5}$ Professora convidada do \\ curso lato sensu em Atenção \\ Farmacêutica, UNIFAL-MG. \\ Endereço eletrônico: laracriz@ \\ yahoo.com.br
}

Recebido em: 11/07/2010 Aprovado em: 21/04/2011 
O processo saúde-doença inclui tanto a dimensão coletiva, já que a saúde dos sujeitos é influenciada pela saúde do contexto no qual estão inseridos, como pela dimensão individual, pois o sofrimento e o adoecimento, embora possam ser compartilhados com outras pessoas, são experiências pessoais e singulares (LACERDA, 2002).

Nas últimas décadas, o interesse da população mundial por práticas nãoconvencionais em saúde (PNCS) vem aumentando substancialmente, estimulando os órgãos gestores da saúde mundial - como a Organização das Nações Unidas para Agricultura e Alimentação (FAO) e a Organização Mundial da Saúde (OMS) - e de diversos países à implementação e ao desenvolvimento de medidas que visem a corresponder aos anseios da sociedade nessa área (TEIXEIRA, 2005; PAGLIARO; LUZ, 2001).

No Brasil, visando corresponder aos anseios e procura pelas práticas nãoconvencionais em saúde (PNCS), em 3 de maio de 2006 entrou em vigor a Portaria n. 971, que aprovou a Política Nacional de Práticas Integrativas e Complementares (PNPIC) no Sistema Único de Saúde, considerando que o Ministério da Saúde entende que as práticas acima citadas compreendem o universo de abordagens denominado pela OMS de Medicina Tradicional e Complementar / Alternativa - MT / MCA, sendo as seguintes: Acupuntura, Homeopatia, Fitoterapia, Termalismo Social / Crenoterapia (BRASIL, 2006).

Juntamente com esse contexto mundial, segundo qual a população busca melhor qualidade de vida, sabe-se que a utilização de medicamentos é um processo complexo com múltiplos determinantes e envolve diferentes fatores. E para que ocorra de modo racional, são influenciados por fatores de natureza cultural, social, econômica e política (PERINI et al., 1999). A Atenção Farmacêutica (AF) é a provisão responsável da farmacoterapia com o objetivo de alcançar resultados definidos que melhorem a qualidade de vida, podendo reduzir os problemas previníveis relacionados à farmacoterapia, sendo muito importante como agente de promoção para o uso racional dos medicamentos (COSENDEY et al., 2000).

\section{Justificativa}

O Estado de Minas Gerais é reconhecido no cenário nacional por sua excelente qualidade de atendimento e investimentos nas unidades básicas de saúde. Em 2008, o então Secretário de Saúde do estado, Marcus Pestana, afirmou que a atenção 
básica é a melhor maneira de oferecer uma saúde contínua e preventiva; assim, ao concentrar investimentos, garante ao cidadão uma porta de entrada ao SUS mais eficiente e acolhedora. Os investimentos já atingem mais de R \$367 milhões, sendo que R 17 milhões foram destinados às equipes de saúde da família (ESFs). Os investimentos reduziram as internações por doenças facilmente controláveis por ações preventivas, como desidratação infantil, desnutrição, bronquite, diabetes e hipertensão. Houve também redução de custos com procedimentos de média e alta complexidade, que são mais onerosos para o Estado (MARQUES, 2008).

A busca pelas práticas terapêuticas não-convencionais (fitoterapia, acupuntura, homeopatia, dentre outras) demonstra a necessidade de reconhecer e refletir a possibilidade do estabelecimento de um diálogo construtivo entre a tradição e a medicina moderna, resultando em melhor qualidade de vida para os pacientes. Finalmente, contribui para as decisões dos profissionais que trabalham na área, através da preservação, acomodação ou repadronização dessas práticas para orientar a participação do paciente no seu processo de busca da saúde (MEDEIROS, 1997).

Eisenberg e colaboradores (apud TEIXEIRA, 2005) estimaram que $42 \%$ da população norte-americana fazem uso de práticas não-convencionais em saúde (PNCS) PNCS, com 629 milhões de consultas anuais (excedendo o número total de consultas do sistema primário de saúde dos Estados Unidos), representando um custo adicional de US $\$ 27$ bilhões à população americana, por não estarem disponíveis nos serviços públicos de saúde e não serem reembolsadas pelas empresas de seguro médico. Devido este cenário, 1998, o Congresso dos Estados Unidos autorizou a transformação Instituto de Medicina Alternativa do Instituto Nacional de Saúde (Office of Alternative Medicine do National Institutes of Health - NIH), ou seja, tornou-se acesso para os usuários dos serviços públicos de saúde, e, após seis anos de funcionamento, no Centro Nacional para Medicina Complementar e Alternativa (National Center for Complementary and Alternative Medicine), promovendo um crescimento exponencial das práticas não-convencionais em saúde (PNCS) no país, devido o aumento do acesso aos serviços oferecidos, e, disponibilizando centenas de milhões de dólares para o desenvolvimento de pesquisas nessas áreas, que hoje no Brasil são denominadas Práticas Integrativas e Complementares (PICS).

A Unidade Básica de Saúde (UBS) de São João da Mata é o único local da cidade que dá acesso a tratamentos e cuidados médicos, pois o município não possui 
hospital nem clínicas médicas para tratamentos mais específicos. Em casos de emergência, os pacientes são encaminhados para os hospitais das regiōes vizinhas.

Há poucos estudos na literatura sobre representações sociais em práticas alternativas e complementares e prática da atenção farmacêutica em unidades básicas de saúde (UBS). Desta forma, é importante o levantamento dessas características, visto que essas informações poderão ser úteis na implementação dessas racionalidades médicas, bem como do programa de atenção farmacêutica nesses locais, beneficiando os usuários. Desta forma, este estudo tem por objetivo investigar o conhecimento e aceitação das terapias integrativas e complementares e atenção farmacêutica por usuários de unidades básicas de saúde do SUS.

\section{Metodologia}

O estudo foi realizado na Farmácia da Unidade Básica de Saúde situada no centro da cidade de São João da Mata, em Minas Gerais, e foi escolhida para a presente pesquisa justamente por se tratar de uma unidade básica de saúde e ser o principal local de acesso aos pacientes/população.

O município de São João da Mata está situado na Região Sul de Minas Gerais, na microrregião de Santa Rita do Sapucaí. Foi fundado como distrito de Silvianópolis em 1943 e emancipado em 1962. Sua distância da capital mineira é de 412 km e de São Paulo é de 290 km. A população residente no ano de 2001, de acordo com a Fundação Instituto Brasileiro de Geografia e Estatística (IBGE), é de 2.752 habitantes, sendo 1.610 habitantes na área urbana e 1.142 habitantes na zona rural (PREFEITURA MUNICIPAL, 2010).

A pesquisa foi elaborada em forma de entrevista semiestruturada, ou seja, um formulário, com base naquele proposto por Lemos et al. (2001). A pertinência da escolha de tal instrumento justifica-se pelo que transparece ao descrever informaçôes que se pode obter: dados que referem às maneiras de atuar, crenças ou comportamentos da população. Foram entrevistados 35 pacientes dentre aqueles que se dirigem semanalmente à Unidade Básica de Saúde para se consultar com médicos de diversas especialidades e, após consulta, retiram seus medicamentos na farmácia básica; ou ainda aquelas pessoas que passaram na farmácia para retirada de medicamentos com o cartão de cadastro dos agentes de saúde. Os pacientes entrevistados concordaram em participar espontaneamente da pesquisa, assinando o termo de consentimento. 
A coleta dos dados foi realizada na primeira quinzena do mês maio de 2009.

A todos os participantes foi garantido o sigilo de seus nomes e também que os dados somente seriam usados pelos pesquisadores. Foi utilizado um formulário com questôes fechadas que foi preenchido pelos pacientes ou por intermédio do farmacêutico para os pacientes que não conseguiam preencher (pediam para o farmacêutico anotar suas opinióes e respostas). Foram coletados dados para a identificação dos sujeitos, como sexo, idade, profissão e crença ou religião. Também a coleta foi composta por questões que visaram a conhecer as percepções dos sujeitos a respeito da aceitação da implantação das terapias alternativas e complementares no SUS e da aceitação da implantação da Atenção Farmacêutica.

A pesquisa foi conduzida dentro de padrões éticos exigidos pela Comissão Nacional de Ética em Pesquisa/Conselho Nacional de Saúde/Ministério da Saúde (CONEP/CNS/MS).

\section{Análise dos dados}

Os dados coletados foram do tipo qualitativo, gerados a partir do registro detalhado no preenchimento do formulário, e foram organizados por categorias para facilitar a interpretação dos resultados.

Para a efetivação das análises foram realizados, primeiramente, momentos de leitura livre dos dados para um conhecimento geral do resultado. Posteriormente, buscou-se identificar os temas mais significativos e recorrentes. A partir dessa identificação, procurou-se organizar os dados em categorias com vistas ao estabelecimento de conexôes entre essas e os objetivos propostos pelo estudo, buscando aumentar a compreensão sobre o tema. Este estudo não verifica o fenômeno em si, mas busca compreender o que os fenômenos representam para as pessoas (MOSCOVICI, 2003).

\section{Resultados e discussão}

\section{Dados quantitativos}

Foi considerada importante, para efeito de análise e discussão, a identificação dos participantes quanto a sexo, religião, grau de escolaridade e o entendimento que eles possuem em sobre a definição do que é Atenção Farmacêutica e o que seriam as terapias alternativas e complementares. Esses dados foram contemplados na primeira parte do formulário. 
Os sujeitos que responderam ao formulário, em sua maioria, eram mulheres (74\%) e católicos (71,42\%). Os demais sujeitos declararam ser evangélicos, crentes e protestantes. Estes dados são corroborados por aqueles obtidos por Rodrigues Neto et al. (2009). Em 2006 e 2007, foi realizada uma pesquisa pelo Datafolha em que foram entrevistados 44.642 sujeitos, dos quais $64 \%$ declararam ser católicos, 17\% evangélicos pentecostais e 5\% pentecostais (DATAFOLHA, 2010). Quanto ao grau de escolaridade, $68,57 \%$ dos entrevistados possuem o primeiro grau incompleto; $23 \%$, segundo grau completo; e $9 \%$, curso superior.

Quando indagados sobre o tema da Atenção Farmacêutica, a maioria dos pacientes não soube responder do que se tratava e somente após explicação clara e simples do assunto fornecida pela pesquisadora puderam responder. Os resultados foram: $45,71 \%$ ouviram falar em AF, $22,85 \%$ afirmaram saber do que se trata e $31,42 \%$ nunca ouviram falar em AF. Isto evidencia a necessidade de maior atuação do farmacêutico dentro dos programas de saúde pública. Quando questionados sobre a implantação da AF, $100 \%$ das mulheres entrevistadas foram receptivas a sua implantação, enquanto $28,57 \%$ dos homens entrevistados não gostariam de ser acompanhados por um farmacêutico em seu tratamento.

A segunda questão do formulário tratava do conhecimento ou aceitação das terapias integrativas e complementares no SUS. Quando indagados sobre as terapias alternativas e complementares, $100 \%$ dos pacientes não souberam responder do que se tratava e, novamente, após explicação clara e simples do assunto pela pesquisadora é que estes escolheram as terapias que aceitariam em seu tratamento (figura 1).

Figura 1: Aceitação das Práticas Alternativas e complementares pelos sujeitos entrevistados.

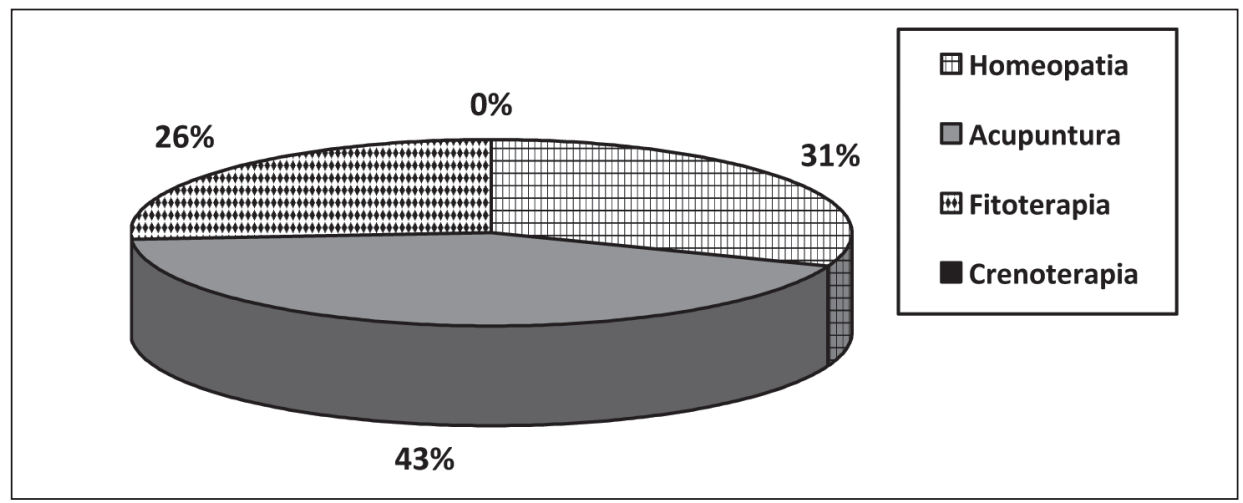


Tabela 1 - Homens e mulheres em relação à aceitação das Terapias Alternativas e

Complementar

\begin{tabular}{l|c|c|c|c}
\hline $\begin{array}{l}\text { Terapias integrativas e } \\
\text { complementares }\end{array}$ & $\begin{array}{c}\text { Homens } \\
\text { (n) }\end{array}$ & $\begin{array}{c}\text { Porcentagem } \\
\%\end{array}$ & $\begin{array}{c}\text { Mulheres } \\
\text { (n) }\end{array}$ & $\begin{array}{c}\text { Porcentagem } \\
\%\end{array}$ \\
\hline Acupuntura & 6 & 66,66 & 21 & 60 \\
\hline Fitoterapia & 3 & 33,33 & 10 & 28,57 \\
\hline Homeopatia & 5 & 55,55 & 8 & 22,85 \\
\hline Crenoterapia/ termalismo & 0 & 0 & 0 & 0 \\
\hline Total & 9 & 100 & 26 & 100 \\
\hline
\end{tabular}

Observa-se, na tabela 1, que vários pacientes apontaram mais de um tipo de terapia integrativa e complementar, sendo que a maioria dos homens optou por Acupuntura e Homeopatia, enquanto a maior parte das mulheres preferiu utilizar Acupuntura e Fitoterapia.

\section{Dados qualitativos}

\section{Aceitação das práticas integrativas e complementares por parte dos prescritores}

Comparando as respostas dadas pela comunidade sãojoanense, indagou-se sobre a aceitação da implantação e indicação a pacientes das terapias alternativas e complementares. Três dos médicos que atendem na unidade básica de saúde onde foi realizada a pesquisa responderam, e suas respostas foram:

Médico 1: Não indicaria a nenhum paciente meu, pois não tenho conhecimento de eficácia e penso que só atrapalharia a clínica e atrasaria o tratamento dos pacientes.

Médico 2: Confesso que não tenho conhecimento de como funciona, talvez por esse motivo não associe este tipo de tratamento aos meus pacientes, mas penso que tudo o que o paciente acredita que será bom para seu tratamento é valido! Só acho que deveria ser mais divulgado.

Médico 3: Não vejo problema, talvez indicasse mais se houvesse uma maior divulgação.

Compararam-se as respostas obtidas pelos prescritores com os resultados de um trabalho sobre a aceitação de práticas não-convencionais em saúde por estudantes de Medicina da Universidade do Sul de Santa Catarina. Nesse estudo, foi demonstrado que o conhecimento dos estudantes de medicina sobre 
acupuntura e homeopatia é muito pequeno ou quase inexistente. Portanto, este dado, ao lado de outros resultados encontrados no estudo, evidencia a necessidade de incluir disciplinas curriculares que abordem as PNCS nos cursos de graduação de Medicina (KÜLKAMP et al., 2007). Por outro lado, em trabalho realizado por Christensen e Barros (2010), foram observadas diferentes formas de inserção das medicinas alternativas e complementares no ensino médico, atitudes positivas dos estudantes de Medicina frente a elas e desejo de aprendê-las com o objetivo de tratar e orientar futuros pacientes.

\section{Expectativa do paciente sobre o que é atenção farmacêutica}

A primeira questão aberta visou a conhecer o que o paciente entende por Atenção Farmacêutica. As respostas foram agrupadas em subcategorias, a saber:

- Atenção/Orientação - ao se analisar as respostas, percebeu-se que alguns dos sujeitos esperam do farmacêutico "atenção", como revelam as seguintes respostas:

Uma pessoa que saiba nos dar atenção como pessoa normal e ouvir nossos problemas.

Atenção com o cliente por que o médico não dá atenção.

Explicar como tomar remédios.

Tratar a gente bem e resolver problema de remédio.

Que ele possa ajudar no controle do paciente que toma mais de um medicamento, para tomar a quantidade certa e horário certo.

Acho que o farmacêutico responde aos questionamentos do paciente com educação e respeito.

As respostas revelam que os pacientes esperam dos farmacêuticos uma relação mais estreita, como forma de amizade, dando uma atenção maior e resposta a dúvidas que outros profissionais não oferecem. Mas, no geral, estão satisfeitos com o atendimento recebido.

- Acompanhamento

É necessário um programa para quem faz tratamento psiquiátrico; a dificuldade de marcação de consulta e exames é grande e, com isso, o paciente tem períodos de regressão, devido à falta de medicamento ou acompanhamento. Os pacientes psiquiátricos são esquecidos pelo SUS e só são atendidos quando entram em surto, e não é isso que vai melhorar a situação deles; é a prevenção e o acompanhamento que podem evitar a internação e a regressão da doença.

Acompanhamento dos remédios; como estão sendo usados pelos pacientes. 
Após explicação sobre o que é Atenção Farmacêutica, seguem-se as respostas dos pacientes em relação à implantação da AF.

Evitaria muitos erros na hora de tomar remédio.

A população terá conhecimento de como tomar remédio.

Importante nos meus tratamentos, ele é que me dá as orientaçōes dos remédios.

Haveria mais entendimento por parte da população.

Uma pessoa de confiança que posso parar e falar os meus problemas.

Tenho um atendimento melhor na farmácia quando atendido por um farmacêutico, ele se interessa pela saúde, não pensa só em vender.

Não precisa de atenção farmacêutica.

Aqui os entrevistados demonstraram um bom relacionamento com os farmacêuticos, estando satisfeitos com a atenção e informação fornecida por esse profissionais. Muitas vezes, o paciente necessita somente de uns minutos de atenção para se sentir melhor e saber que está sendo assistido por um profissional da saúde. De acordo com Duque e Lourenço (2006), o que se pode perceber é que as pessoas não querem somente profissionais para orientar sobre o uso dos medicamentos; eles querem uma pessoa simpática, que converse com ela de maneira clara, ouça seus problemas e aconselhe, demonstre de alguma forma que a saúde dela é importante e estabeleça uma relação de confiança duradoura.

\section{Conclusões}

Esta pesquisa demonstra que a grande maioria dos pesquisados aceitaria o uso de terapias alternativas e complementares se estas fossem oferecidas pela unidade de saúde de São João da Mata-MG, além de acharem importante uma maior atenção do farmacêutico no seu atendimento. No entanto, pode-se observar que ainda faltam divulgação e programas sociais que busquem apresentar ao público, principalmente aos profissionais de saúde - em especial aos de medicina -, os benefícios que tais terapias podem trazer para a vida dos pacientes.

Assim sendo, pode-se dizer que a Política de Práticas Integrativas e Complementares, regulamentada pela Portaria 971 de , tem tudo para dar certo no atendimento básico de saúde em São João da Mata-MG, e que a Atenção 
Farmacêutica e as Terapias Alternativas e Complementares serão estratégias de cuidado da saúde importantes para a população que é carente de cuidados e necessita do atendimento público de saúde.

\section{Referências}

CHRISTENSEN, M.C.; BARROS, N.F. Medicinas alternativas e complementares no ensino médico: revisão sistemática. Rev Bras de Educ Méd, v. 34, n. 1, p. 97-105, 2010.

COSENDEY, M.A.E. et al. Assistência farmacêutica na atenção básica de saúde: a experiência de três estados brasileiros. Cad. Saúde Pública, c. 16, n. 1, p. 171-182, jan 2000. DATAFOLHA. 64\% dos brasileiros se declaram católicos. São Paulo, 16 abril de 2007. Disponível em: http://datafolha.folha.uol.com.br/po/ver_po.php?session=447, Acesso em: 25 abr 2010.

DUQUE, D.C.C.; LOURENÇO, E.B. Relaçāo Farmacêutico-paciente: um novo olhar. 2006. Trabalho de conclusão de curso de Especialização em Atenção Farmacêutica. Universidade Federal de Alfenas. Disponível em: http://www2.unifal-mg.edu.br/gpaf/?q=node/3, Acesso em: 08 jul 2010.

KÜLKAMP, I.C. et al. Aceitação de práticas não-convencionais em saúde por estudantes de medicina da Universidade do Sul de Santa Catarina. Rev. bras. educ. med., v. 31, n. 3, p. 229-235, dez 2007.

LACERDA, A. Apoio social e a concepção do sujeito na sua integração entre corpo-mente: uma articulação de conceitos no campo da Saúde Pública. 2002. 94f. Dissertação (Mestrado em Endemias, Ambiente e Sociedade) - Escola Nacional de Saúde Pública, Fundação Oswaldo Cruz, Rio de Janeiro, 2002.

LEMOS, J.Z.; BATISTA, T.A.; CANCIAN, T.A. Terapias alternativas sob o olhar dos alunos graduandos em enfermagem. Revista do Centro Universitário Barão de Mauá, v. 1, n. 2, jul-dez 2001. Disponível em: http://www.baraodemaua.br/comunicacao/publicacoes/ jornal/vln2/artigo07.html. Acesso em: 10 jan 2010.

MARQUES, M. Secretário de saúde do Ceará conhece ações mineiras na área. Jusbrasil, dez. 2008. Disponível em: <www.jusbrasil.com.br/noticias>. Acesso em: 10 jan. 2010.

MEDEIROS, L.S. Práticas terapêuticas não-convencionais usadas por idosos. 1997. 197f. Tese (Doutorado em Saúde Pública) - Universidade de São Paulo, Faculdade de Saúde Pública, São Paulo, 1997.

BRASIL. Ministério da Saúde. Portaria n. 971, de 3 de maio de 2006. Aprova A Política Nacional de Práticas Integrativas e Complementares (PNPIC) no Sistema único de Saúde Diário oficial da Uniāo, Brasília, n. 84, seção I p. 19, 04 maio 2006. 
MOSCOVICI, S. Representaçôes sociais: investigação em psicologia social. Petrópolis:

Vozes, 2003.

PAGLIARO, G.; LUZ, H. Política Nacional de Práticas Complementares e Integrativas: o caso da Homeopatia. Rio de Janeiro: ABMH. Disponível em <http gemhca.org.br/divulgação. htm>. Acesso em: 25 maio 2007.

PERINI, E. et al. Consumo de Medicamentos e adesão às prescrições: objeto e problema de epidemiologia. Rev. Ciênc. Farm. v. 20, p. 471-488, 1999.

PREFEITURA MUNICIPAL DE SÃO JOÃO DA MATA-MG. Site oficial. Onde estamos. Disponível em: http://www.sjmata.com.br/index.html, Acesso em: 26 abr 2010.

RODRIGUES-NETO, J.F.; FARIA, A.A.; FIGUEIREDO, M.F.S. Medicina complementar e alternativa: utilização pela comunidade de Montes Claros, Minas Gerais. Rev Assoc Med Bras, v. 55, n. 3, p. 296-30, 2009.

TEIXEIRA, Z.M. A ciência das formas peculiares de curar. Jornal da USP. 2005; 20 (718). Disponível em: http://www.usp.br/jorusp/arquivo/2005/jusp718/pag0405.htm. Acesso em: 26 jan 2010. 
Pharmaceutical care and complementary and alternative medicine in the SUS: Knowledge and acceptance on the part of the population from the city of São João da Mata, Minas Gerais state, Brazil

In 2006, the Ministry of Health created the National Policy on Integrative and Complementary Therapies in the Unified Health System. This study aimed to investigate the knowledge and acceptance of complementary and integrative therapies and pharmaceutical care by SUS users. The study was conducted in the pharmacy of a primary healthcare unit in São João da Mata city, Minas Gerais state, Brazil. A cross sectional study was performed qualitatively and quantitatively with three doctors and 35 service users. Of these, $100 \%$ did not know the complementary and integrative therapies. After clear and simple explanation by the researcher, $31.42 \%$ said they would accept the use of herbal medicine, $51.42 \%$ accepted acupuncture, $37.14 \%$ would use homeopathy and none would use crenotherapy. When asked about the pharmaceutical care, $45.71 \%$ said they had heard of this matter, $22.85 \%$ know what it is and $31.42 \%$ had never heard of it. When the three doctors who at the clinic were questioned, there was indifference, non-acceptance and acceptance, respectively. In conclusion, this study showed that the vast majority of respondents would accept the complementary and integrative therapies if they were offered by the health unit. In addition, users find important a greater role of the pharmacist. One need to implement outreach programs for patients and especially for prescribers of complementary and integrative practices.

> Key words: pharmaceutical care, complementary and alternative medicines, acceptance. 